\title{
Swallowing-induced atrial tachycardia
}

\author{
Rebecca Mathew MD, Martin S. Green MD, Pablo B. Nery MD
}

Cite as: CMAJ 2018 April 23;190:E507-9. doi: 10.1503/cmaj.171261

A n 87-year-old woman presented at the emergency department with sudden and recurring episodes of lightheadedness that occurred while she was eating and drinking. The patient did not feel any chest pain or heart palpitation, and she had not experienced fainting, gastrointestinal distress, difficulty with or painful swallowing, nausea or heartburn. She had a history of rheumatic fever and bacterial endocarditis, which had been treated with a prolonged course of antibiotics given intravenously when she was an adolescent.

On initial assessment, the patient was hemodynamically stable, with no substantial orthostatic change. The results of cardiac, respiratory and gastrointestinal examinations were normal. A gross neurologic screen was unremarkable. Laboratory investigations, including cell count and differential, serum chemistry, extended electrolytes, liver panel, coagulation profile and cardiac biomarkers, as well as chest radiography, were normal.

An initial electrocardiograph (ECG) showed normal sinus rhythm with first-degree atrioventricular (AV) block (PR interval $236 \mathrm{~ms}$ ) (Figure 1). Cardiac monitoring within the first hour of presentation showed reliable correlation between each episode of swallowing and subsequent onset of a rapid, narrow complex
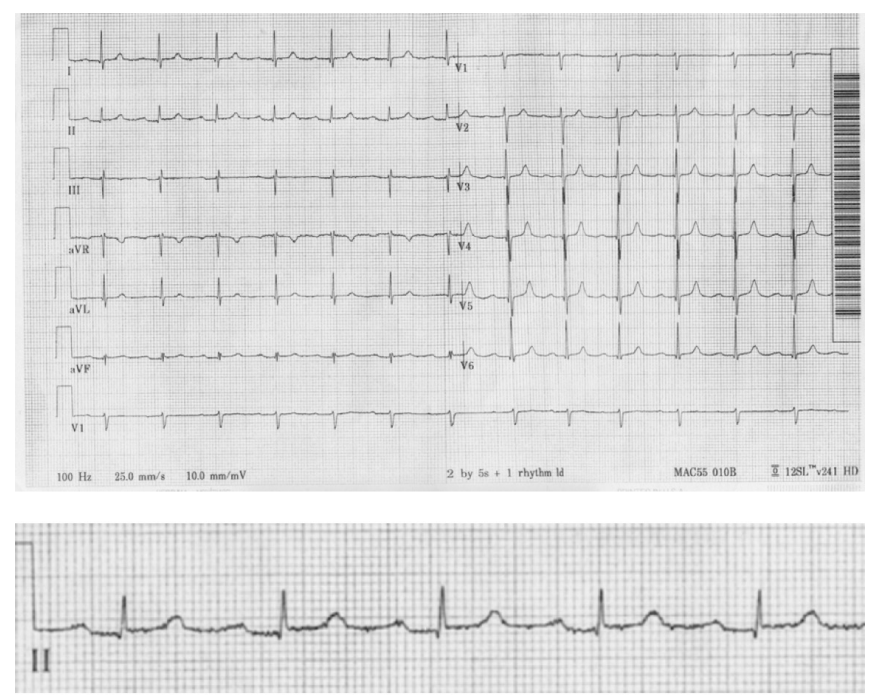

Figure 1: Initial 12-lead electrocardiogram (and magnified view of lead II) from an 87-year-old woman showing normal sinus rhythm with firstdegree atrioventricular block (PR interval $236 \mathrm{~ms}$ ). The patient had presented with sudden and recurring episodes of lightheadedness that occurred while she was eating and drinking.

\section{KEY POINTS}

- Swallowing-induced atrial tachycardia is an uncommon atrial tachyarrhythmia, most commonly seen in middle-aged men who have structurally normal hearts and gastrointestinal systems.

- Diagnosis is made with symptom-arrhythmia correlation, often with Holter monitoring.

- Conservative therapy is usually effective, involving the swallowing of smaller food boluses, the avoidance of triggering foods or pharmacologic therapy with $\beta$-blockers or calciumchannel blockers.

- Patients with refractory cases can undergo radiofrequency catheter ablation.

tachycardia. Between episodes of swallowing or eating, the patient remained in normal sinus rhythm with no evidence of atrial fibrillation or flutter. Each episode of tachyarrhythmia resulted in presyncope, similar that which had lead to her presentation. Serial ECGs taken during tachyarrhythmia showed atrial tachycardia with 3:2 AV conduction (Figure 2), followed by atrial tachycardia with 1:1 AV conduction at a rate of 180 beats/ min (Figure 3).
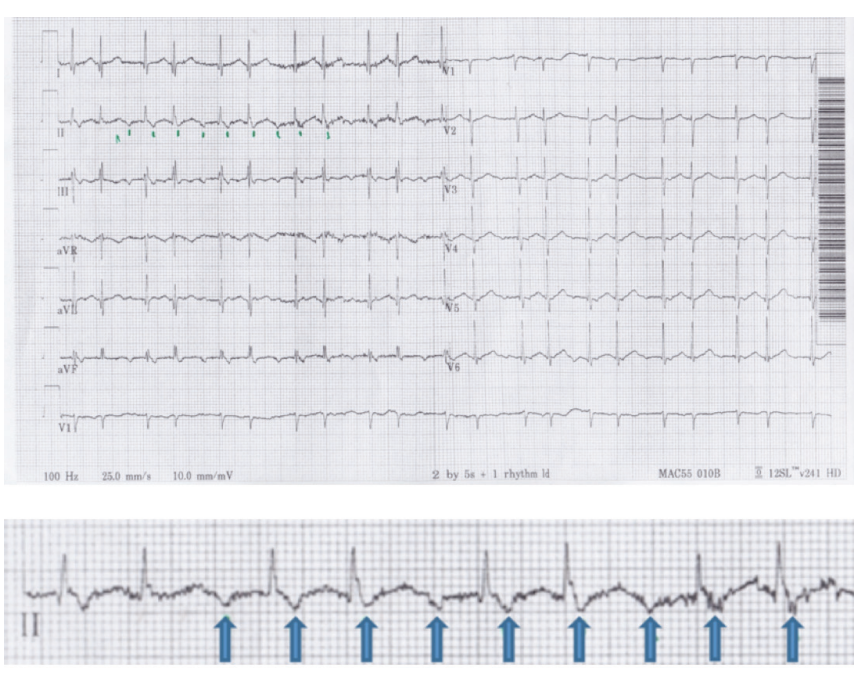

Figure 2: A subsequent 12-lead electrocardiogram (and magnified view of lead II) taken during tachyarrhythmia showing atrial tachycardia with 3:2 conduction. Arrows indicate negative $\mathrm{P}$ waves. 

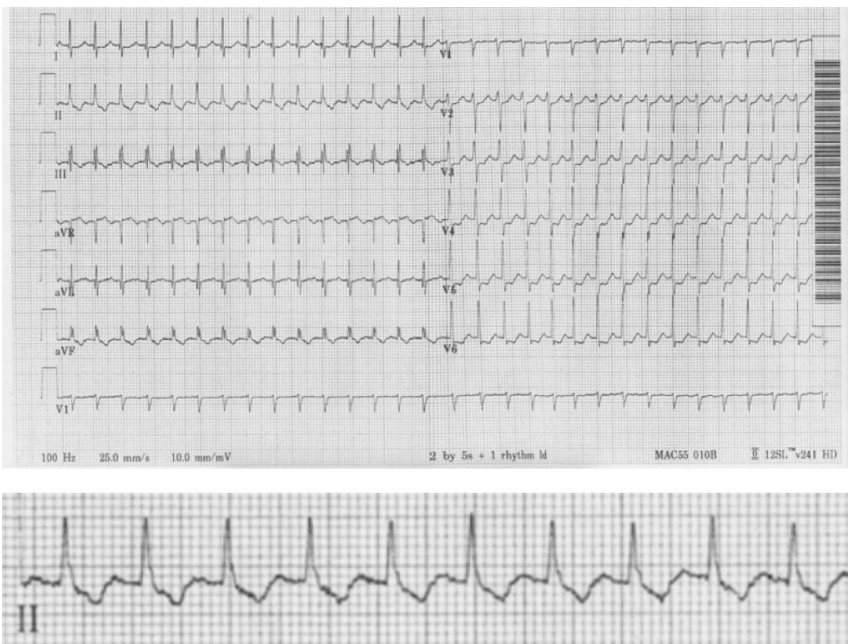

Figure 3: A subsequent 12-lead electrocardiogram (and magnified view of lead II) taken during tachyarrhythmia showing atrial tachycardia with 1:1 conduction at 180 beats/min.

Before receiving any treatment, the patient's symptoms began to decrease in severity and frequency, and she was able to drink liquids without having presyncope. A transthoracic echocardiogram showed preserve biventricular size and function with no valvular abnormalities - specifically, no evidence of rheumatic heart disease. The patient was started on a low-dose $\beta$ blockade (metoprolol, $25 \mathrm{mg}$ twice daily, taken orally). After her first dose, the patient showed complete resolution of her symptoms, and she was able to eat breakfast without presyncope or tachyarrhythmia on telemetry. The patient's condition was monitored in the emergency department for an additional 24 hours to ensure complete symptom resolution. She was discharged home in stable condition. At 12 weeks' follow-up in clinic, she remained symptom-free and was tolerating treatment with no adverse effects.

\section{Discussion}

Swallowing- or deglutition-induced atrial tachycardia is an uncommon condition, occurring in about $0.6 \%$ of patients who present with paroxysmal atrial arrhythmias. ${ }^{1}$ Most cases of presyncope and syncope with swallowing are associated with bradyarrhythmias. Swallowing-induced bradyarrhythmias are thought to be vagally mediated and are often associated with underlying structural abnormalities of the heart (i.e., ischemic heart disease), gastrointestinal system (i.e., diffuse esophageal spasm, esophageal diverticulum, achalasia) or both. Swallowing-induced atrial tachycardia was first described by Sakai and Mori in 1926 as schlucktachycar$d i e,{ }^{2}$ and only about 50 cases have been reported in the literature to date. ${ }^{1}$ In contrast to swallowing-induced bradyarrhythmias, swallowing-induced atrial tachycardias are more commonly associated with structurally normal hearts and gastrointestinal systems. They also tend to occur in men older than 35 years of age, often between the fifth and sixth decades of life. ${ }^{1}$

\section{Mechanisms}

Swallowing-induced atrial tachycardias have three commonly postulated mechanisms. ${ }^{3}$ First, tachyarrhythmias begin after mechanical stimulation of the left atrium by a food bolus in the distended esophagus. Cohen and colleagues showed the plausibility of this mechanism by reproducing atrial arrhythmias by inflating an intraluminal esophageal balloon at the subcarinal level. ${ }^{4}$ However, their findings have not been reproducible, and because swallowing-induced atrial tachycardias can be induced with dry swallowing alone, this mechanism likely does not account for all cases. Tada and colleagues reported three cases of swallowing-induced atrial tachycardias with subsequent electrophysiological studies that suggested the origin to be in the right pulmonary veins or right atrium, which is not adjacent to the esophagus, meaning that the mechanism of action was not direct mechanical stimulation. ${ }^{1}$

Second, swallowing-induced atrial tachycardias may be a consequence of a vagal reflex initiated by deglutition, because primary peristalsis in the esophagus is mediated by centrally activated firing of vagal efferent fibres. Vagal stimulation can shorten the refractory period of atrial tissue in a nonuniform manner, with repolarization dispersion resulting in microreentrant circuits triggered by a premature atrial contraction. ${ }^{3,5}$ However, vagolytic agents, such as atropine, do not consistently suppress atrial tachycardia, strengthening arguments against a vagal reflex.

Finally, swallowing may stimulate an adrenergic reflex in the esophagus. Activation of the sympathetic nervous system can result in asynchronous atrial depolarization and trigger atrial activity through delayed depolarizations. Again, this nonuniform atrial depolarization can result in focal reentry, leading to atrial arrhythmias. ${ }^{6,7}$

\section{Diagnosis}

Diagnosis can be made based on reliable and consistent symptom documentation and exclusion of other mechanisms of tachyarrhythmia; we suggest using Holter or loop monitor with patient feedback. We suggest that biochemical abnormalities be ruled out with baseline laboratory investigations (including complete blood count with differential, renal function tests, electrolytes, calcium, phosphate, magnesium, thyroidstimulating hormone, cardiac biomarkers and pro b-type natriuretic peptide, if available). In addition, a baseline radiograph of the chest can be completed. In our experience, because swallowing-induced atrial tachycardias are most commonly seen in patients with normal cardiac anatomy, an echocardiogram can ensure that there are no structural abnormalities. Gastroscopy to look for underlying esophageal disorders has been included in the diagnostic evaluation in several case reports; however, the literature to date cites that a structurally normal gastrointestinal tract is most common. ${ }^{7}$

\section{Management}

Initial management is conservative, with lifestyle changes. ${ }^{8}$ Tachyarrhythmias can be prevented by avoiding triggering foods; patients are advised to minimize their intake of caffeine, alcohol and cool liquids, and to consume chopped foods to minimize the swallowing of large boluses. For patients with persistent symptoms despite lifestyle changes, several classes of medication 
have been used with variable success. The most commonly used medications include $\beta$-blockers, calcium-channel blockers and class IA, IC and III antiarrhythmic agents.

Radiofrequency catheter ablation can provide a permanent solution for patients with refractory cases., ${ }^{1,7,9}$ During the procedure, tachyarrhythmia may be reproduced with dry or wet swallowing, with concurrent mapping to identify the atrial site of origin. Radiofrequency catheter ablation is particularly useful when patients have reliable reproducibility with a single arrhythmogenic focus. ${ }^{7,9}$ Surgical intervention has been used historically, with interpleural repositioning of the esophagus to allow separation from the left atrium; ${ }^{10}$ however, a surgical approach is no longer considered relevant.

\section{References}

1. Tada H, Kaseno K, Kubota S, et al. Swallowing-induced atrial tachyarrhythmias: prevalence, characteristics, and the results of the radiofrequency catheter ablation. Pacing Clin Electrophysiol 2007;30:1224-32.

2. Sakai D, Mori F. Uber einen Fall von Sog "Schluck-tachykardie". Z Gesamte Exp Med 1926;50:106-9.

3. Suarez LD, Chiozza MA, Foye R, et al. Swallowing-dependent atrial tachyarrhythmias. Their mechanism. J Electrocardiol 1980;13:301-5.

4. Cohen L, Larson D, Strandjord N. Swallowing induced atrial fibrillation [abstract]. Circulation 1970;26(Suppl 3):145.

5. Alessi R, Nusynowitz M, Abildskov JA, et al. Nonuniform distribution of vagal effects on the atrial refractory period. Am J Physiol 1958;194:406-10.
6. Kanjwal Y, Imran N, Grubb B. Deglutition-induced atrial tachcardia and atrial fibrillation. Pacing Clin Electrophysiol 2007;30:1575-8.

7. Undavia M, Sinha S, Mehta D. Radiofrequency ablation of swallowing-induced atrial tachycardia: case report and review of literature. Heart Rhythm 2006; 3:971-4.

8. Tanoue K, Sonoda M, Yamasita E, et al. Swallowing induced atrial tachyarrhythmia triggered by solid foods. Circulation 2014;130: e113-5.

9. Yamauchi Y, Aonuma K, Sekiguchi Y, et al. Curative therapy for swallowinginduced tachycardia by pulmonary vein antrum isolation. J Cardiovasc Electrophysiol 2005;16:1370-4.

10. Burton JR, Sachs HJ, Keon WJ, et al. Intrapleural positioning of esophagus for treatment of swallowing-induced arrhythmia. Chest 1981;79:367-8.

\section{Competing interests: None declared.}

This article has been peer reviewed.

The authors have obtained patient consent.

Affiliations: Adult Cardiology Residency Program (Mathew); Arrhythmia Service (Green, Nery), Division of Cardiology, University of Ottawa Heart Institute, Ottawa, Ont.

Contributors: All of the authors participated in the conception, design, and writing and revision of the article, and the analysis and interpretation of the data. All of the authors approved the final version of the manuscript to be published and agreed to act as guarantors of the work.

Correspondence to: Rebecca Mathew, rmathew@ottawaheart.ca 